\title{
La Universidad de Buenos Aires bajo la dictadura. Una aproximación a través del estudio del perfil, discurso y pronunciamientos públicos de dos de sus rectores
}

\author{
The University of Buenos Aires under the Dictatorship. \\ An Approach through the Study of the Profile, Speech \\ and Public Pronouncements of two of its Rectors
}

\author{
Pablo Buchbinder* \\ Universidad Nacional General Sarmiento, Argentina
}

http://dx.doi.org/10.20318/cian.2016.3435

Recibido: $\quad 04 / 12 / 2015$

Aceptado: $\quad 14 / 10 / 2016$

Resumen: El propósito de este artículo consiste en analizar los discursos de dos de los Rectores de la Universidad de Buenos Aires -Alberto Rodríguez Varela y Lucas J. Lennon- durante la Dictadura Militar iniciada el 24 de marzo de 1976 y finalizada el 10 de diciembre de 1983. Lucas J. Lennon fue Rector entre noviembre de 1978 y noviembre de 1981. Alberto Rodríguez Varela fue rector entre noviembre de 1981 y diciembre de 1982. En el artículo se focaliza especialmente en el perfil académico de los personajes, en su perspectiva de las políticas universitarias y en las estrategias para vincular a la universidad con otras instituciones educativas, políticas y culturales. También se subrayan los intentos de despolitizar la vida universitaria.

Palabras clave: Argentina, universidad, dictadura, catolicismo.
Abstract: The purpose of this article is to analyze the speeches of two of the Rectors of the University of Buenos Aires -Lucas J. Lennon and Alberto Rodríguez Varela- during the military dictatorship that began in 1976 and ended in 1983. Lennon served as rector between November 1978 and November 1981 and Rodriguez Varela from November 1981 to December 1982. The article focuses especially on the academic profile of the Rectors, on the perspectives of university policies and on the strategies to link the university with other educational, political and cultural institutions. Attempts to avoid the politicization of university life are also highlighted in the article.

Keywords: Argentina, university, dictatorship, catholicism.

*pbuchbin@ungs.edu.ar 


\section{Introducción}

El propósito de este trabajo consiste en ofrecer una aproximación a la historia de la Universidad de Buenos Aires bajo la dictadura militar iniciada en 1976 a partir del estudio del perfil, pensamiento y pronunciamientos públicos de dos de los funcionarios que ocuparon el rectorado en esa etapa: Lucas J. Lennon y Alberto Rodríguez Varela. El primero de ellos ejerció el cargo de Rector entre el 24 de noviembre de 1978 y el 20 de noviembre de 1981. El segundo lo reemplazó hasta el 28 de diciembre de 1982. Se trata de un período de poco más de cuatro años que fue, además, el de la consolidación y afirmación en el poder del llamado "Proceso de Reorganización Nacional". Hacia el año 1978, efectivamente, la Dictadura había terminado por asegurar su poder y dominio sobre el conjunto de la sociedad argentina eliminando los principales atisbos públicos de disconformidad y resistencia ${ }^{1}$. Cabe destacar aquí, sin embargo, que el régimen encontró dificultades significativas a la hora de asegurar un gobierno estable para la Universidad de Buenos Aires, a diferencia de lo que ocurrió por ejemplo en la Universidad Nacional de La Plata que, durante casi todo el período dictatorial, contó con un único Rector, Guillermo Gallo. El primer Rector designado en julio de 1976 luego de una breve intervención militar, el ingeniero Alberto Constantini, renunció dos meses después de asumir

\footnotetext{
${ }^{1}$ Visiones generales sobre la dictadura pueden verse entre otros en Marcos Novaro y Vicente Palermo, La Dictadura Militar, 1976-1983 (Buenos Aires: Paidós, 2001); Hugo Quiroga, El tiempo del proceso (Rosario: Homo Sapiens, 2004), Paula Canelo, El Proceso en su laberinto (Buenos Aires: Prometeo, 2008) y Juan Suriano (Director) Dictadura y Democracia (Buenos Aires: Sudamericana, 2005). La literatura sobre los aspectos educativos bajo la Dictadura en particular los universitarios ha crecido en los últimos años. Específicamente sobre la Universidad bajo la Dictadura pueden verse los textos de Carolina Kaufmann (editora), Dictadura y Educación, Tomo I y II (Buenos Aires: Miño y Dávila, 2001), Pablo Perel, Eduardo Raíces y Martín Perel, Universidad y Dictadura (Buenos Aires: Ediciones del CCC, 2006). Imprescindibles en la consulta son los trabajos de Laura Rodríguez, Católicos, nacionalistas y políticas educativas en la última Dictadura (Rosario: Prehistoria, 2011) y Laura Rodríguez y Germán Soprano, "La política universitaria de la Dictadura Militar en la Argentina: proyectos de reestructuración del sistema de educación superior", en Nuevo Mundo-Mundos Nuevos (2009), consultable en nuevomundo.revues.org/56023. Una breve y contemporànea síntesis de lo acaecido en el sistema universitario bajo la Dictadura durante su primer año en Sara Pallma, "Un año en la Universidad", en Perspectiva Universitaria, N 2, (1977): 61-85. Un caso particular en Gabriela Aguila, "La Universidad Nacional de Rosario en Dictadura”, en Polhis, N 17 (2015): 146-178. Una perspectiva clásica sobre la situación general del sistema educativo en esos años puede verse en el texto de Juan Carlos Tedesco, Cecilia Braslavsky y Ricardo Carciofi, El proyecto educativo autoritario 1976-1983 (Buenos Aires: FLACSO, 1985). Una visión más reciente en Pablo Pineau, "Reprimir y discriminar. La educación en la última dictadura cívicomilitar(1976-1983), en Educar em Revista, 51(2014:103-122).
} 
a raíz de diferencias con los criterios generales de la política universitaria sostenida por el Ministro de Cultura y Educación. Su sucesor, Luis Cabral, nombrado en febrero de 1977, dejó el cargo en agosto del año siguiente. Las diferencias con las autoridades nacionales y en particular con los Ministros a cargo del área de Educación remitían, en ambos casos, a criterios en torno a las formas en que debía avanzarse en los procesos de institucionalización de la casa de altos estudios y en el margen de autonomía que ésta debía conservar. Las gestiones de Lennon y Rodríguez Varela se caracterizaron en cambio por una mayor sintonía con lo que las autoridades militares esperaban de los gobiernos universitarios, en particular del de la UBA, institución rectora del sistema por su prestigio, el número de alumnos y por el impacto a nivel público y nacional de los conflictos que en ella se suscitaban. Diversos autores, por otra parte, han subrayado la importancia que el régimen militar le otorgó a las políticas educativas y particularmente a las universitarias dentro de sus estrategias de control y represión. Como han destacado Marcos Novaro y Vicente Palermo, entre otros, las instituciones educativas fueron identificadas como ámbitos especialmente peligrosos a los que había que neutralizar².

Este artículo está basado en un primer término en los discursos y pronunciamientos públicos de ambos funcionarios tal como fueron registrados y seleccionados por la Secretaría de Prensa y Difusión dependiente del Rectorado de la Universidad de Buenos Aires. Pero estas fuentes han sido luego complementadas con información de periódicos y revistas. Por otra parte, hemos dividido el texto en cuatro secciones. En la primera estudiamos el perfil y la evolución pública y académica de ambas figuras, en la segunda, analizamos su concepción general del orden político que sostenía, en ambos casos, las ideas en torno a la Universidad. En tercer lugar, estudiamos su visión de las políticas universitarias implementadas por el gobierno nacional durante estos años y, por último, nos centramos en las estrategias a partir de las que procuraron vincular a la universidad con otras instituciones educativas y culturales, así como con figuras destacadas en el espacio de la sociedad civil y el estado.

\section{Lucas Lennon y Alberto Rodríguez Varela: breve síntesis de su trayectoria}

Debe notarse que, a diferencia de lo sucedido en otras áreas del estado como las relacionadas con la mayoría de los ministerios o las empresas públicas donde la hegemonía de los funcionarios militares fue evidente, el área de

\footnotetext{
${ }^{2}$ Novaro y Palermo, La Dictadura Militar, 117.
} 
educación, como las de economía y justicia, fue confiada a funcionarios civiles como son los estudiados aquí3. Debe destacarse entonces que Lennon y Rodríguez Varela compartían ámbitos profesionales y de sociabilidad 4 . Compartieron también espacios de ejercicio de la docencia y la actividad universitaria. Ambos eran abogados y cumplieron un papel destacado en el Gobierno Militar mas allá de las funciones desempeñadas en el Rectorado de la UBA. Rodríguez Varela ocupó el Ministerio de Justicia entre el 6 de noviembre de 1978 y el 29 de marzo de 1981. Fue justamente Lennon quien lo reemplazó en ese cargo produciéndose así el intercambio de funciones entre ambos. Previamente, los dos habían desarrollado una extensa carrera en el Poder Judicial. Lennon había ingresado a la Justicia Nacional, en el Fuero Penal, en 1948. Había sido Secretario de Cámara, Juez de Primera Instancia en lo Criminal y Juez de la Cámara Nacional de Apelaciones en lo Criminal y Correccional hasta noviembre de 1973. Había presidido el Tribunal de Enjuiciamiento para magistrados judiciales de la Provincia de Buenos Aires en 1977 y fue luego Conjuez de la Corte Suprema de la Nación entre 1978 y 1979. Por su parte, Rodríguez Varela había comenzado su carrera como Secretario de la Justicia Nacional de Primera Instancia en lo Criminal continuando luego como Secretario de la Cámara Nacional de Apelaciones en lo Criminal y Correccional y Secretario del Tribunal de Enjuiciamiento para Magistrados Nacionales de la Capital Federal. Más tarde, había pasado a desempeñar tareas en la esfera de la Corte Suprema de la Nación. Había sido Secretario Letrado y después Conjuez, como Lennon, de la misma, desarrollando funciones allí entre 1969 y 1981.

\footnotetext{
${ }^{3}$ Rodríguez, Católicos, nacionalistas, 137. Debe notarse que en el caso del Poder Judicial, al que están estrechamente vinculados los dos personajes analizados aquí, se produjo también un intento de renovación a partir del año 1973 que fue dejado sin efecto con el golpe de estado. Diversos autores han subrayado además el fuerte compromiso posterior de sectores relevantes de la magistratura con el orden impuesto por la Dictadura, al respecto puede verse Sofía Lanzilotta y Lucía Castro Feijóo, Justicia y Dictadura (Buenos Aires: Ediciones del CCC, 2014)

${ }^{4}$ Los datos biográficos de ambas figuras fueron extraídos, casi en su totalidad, de "Antecedentes del Doctor Lucas Jaime Lennon y Curriculum Vitae": y "Alberto Rodríguez Varela. Currículum Vitae" ambos publicados en el sitio "Rectores de la Universidad de Buenos Aires entre 1974 y 1983: Currícula Vitae, discursos y comunicados de prensa. Estudio preliminar de Guadalupe Seia", en http://www.uba.ar/historia/contenidos.php? Id-6\&-54. Lucas Jaime Lennon, Documento 1 y Alberto Rodríguez Varela, Documento 1. Consultado en Julio de 2015. La mayor parte de los documentos utilizados en este trabajo difundidos originalmente por la Secretarìa de Prensa y Difusión de la Universidad de Buenos Aires han sido publicados en este sitio que forma parte de la página web del Programa de Historia y Memoria de la Universidad de Buenos Aires.
} 
Paralelamente a sus tareas judiciales habían llevado a cabo una extensa carrera universitaria que atravesó períodos de gobierno militar con otros constitucionales. En principio los dos ocuparon cargos en la Facultad de Derecho de la UBA. Lennon había sido profesor adjunto de Derecho Penal y Rodríguez Varela contaba con una larga trayectoria en la Cátedra de Derecho Político comenzando como jefe de trabajos prácticos por concurso en 1962 y siguiendo luego como profesor adjunto entre 1963 y 1973. En esta Facultad ambos habían desempeñado cargos de gestión. Lennon había sido designado Decano en marzo de 1977. Rodríguez Varela había sido Secretario de Asuntos Académicos entre 1969 y 1971 y luego Decano hasta marzo de 1973, ambos cargos durante el gobierno militar de la autodenominada "Revolución Argentina". Los dos habían sido expulsados de la Facultad a lo largo de ese último año con el ascenso del tercer peronismo. Cabe recordar aquí que la política universitaria en aquel primer tramo de ese gobierno estuvo bajo la dirección de sus grupos más radicalizados de izquierda y que procuró desplazar, entre otros, a aquellos docentes relacionados con el régimen dictatorial iniciado en 1966 o que cumplieron funciones jerárquicas en empresas multinacionales. Rodríguez Varela había retomado su cargo en 1976 ya en tiempos de la Dictadura del llamado "Proceso de Reorganización Nacional".

Los dos desarrollaron también, y en forma paralela a sus trayectorias en el poder judicial y en el espacio universitario público, extensas carreras en el ámbito universitario privado. En principio mantuvieron una estrecha vinculación con la Universidad Católica Argentina, institución de dependencia pontificia. Lennon había sido allí Profesor Titular de Derecho Penal. Por otra parte, Rodríguez Varela fue también aquí Profesor Titular de Derecho Político, cátedra en la que había comenzado en 1962 como auxiliar docente. Además, había sido previamente profesor de la misma asignatura en la Universidad del Salvador vinculada con la orden de los jesuitas. Los dos mantenían estrechos vínculos académicos, además, con el Museo Social Argentino.

Por último, debemos recordar que la especialidad de Lennon era el Derecho Penal y la cuestión de la minoridad mientras que los intereses académicos de Rodríguez Varela eran más diversos. En este sentido, si bien en sus respectivos campos sus textos y trabajos constituían fuentes de referencia tanto para estudiantes como para profesionales, podría afirmarse que la obra de Rodríguez Varela tenía mayor alcance y repercusión y su prestigio académico dentro de los círculos universitarios dedicados al Derecho era probablemente más amplio que el de Lennon. Cabe señalar que Rodríguez Varela había incursionado en la Historia Política y de las Ideas de los siglos 
XIX y XX pero su principal especialidad era el Constitucionalismo y el Derecho Político ${ }^{5}$.

En este sentido, consideramos que un análisis de las perspectivas con las que figuras como Lennon y Rodríguez Varela examinaron los problemas universitarios no puede desvincularse de sus aproximaciones más generales al orden político y social construido en la Argentina durante el siglo XX. En el caso de Lennon esta reconstrucción resulta un tanto más compleja porque su obra es más limitada y constreñida fundamentalmente a textos de naturaleza jurídica en los que prima también una perspectiva fuertemente técnica.

Es más sencilla, en cambio, la aproximación a estas dimensiones del pensamiento en el caso de Rodríguez Varela. Este cuenta con una obra más extensa y referida a aspectos diversos de la Historia Argentina. Sus escritos han sido objeto de análisis y estudio en cursos de Historia Constitucional y Derecho Político desde los años sesenta hasta la actualidad tanto en universidades públicas como privadas. De todas formas, una dificultad adicional para el estudio del pensamiento de Rodríguez Varela desde esta obra reside en el hecho de que la mayor parte de sus trabajos han sido en colaboración sin que se consigne la parte que corresponde a cada autor. De todas formas creemos que, del análisis de algunos de estos fragmentos, en particular de la varias veces reeditada Historia Política y Constitucional Argentina que publicara junto a Ambrosio Romero Carranza y Eduardo Ventura es posible inferir algunos rasgos centrales de las perspectivas con que examinaba la evolución histórica argentina 6 .

En principio, como era previsible, el texto contiene una defensa vehemente del papel de los sectores vinculados a la Iglesia Católica en el desarrollo histórico argentino en el largo plazo. La visión de la Argentina, pensada como "Nación Católica", constituye una dimensión que informa toda la obra. En lo que respecta específicamente a las lecturas de la Historia Contemporánea del país una profunda perspectiva antiperonista se sostiene en las distintas ediciones. La "vocación totalitaria" del primer gobierno de Perón constituye una variable central de la interpretación de esta etapa de la Historia Argentina. Perón se mantuvo, se sostiene en el texto, en base al uso discre-

5 "Antecedentes del Doctor Lucas Jaime Lennon y Curriculum Vitae": y "Alberto Rodríguez Varela. Currículum Vitae" en "Rectores de la Universidad de Buenos Aires entre 1974 y 1983...

${ }^{6}$ Ambrosio Romero Carranza, Alberto Rodríguez Varela y Eduardo Ventura, Historia Política y Constitucional Argentina (Buenos Aires: Ediciones del Círculo Militar, 1992). Véase también las ediciones de 1977, 1981, 1983, y 1991. Debe recordarse también aquí que Eduardo Ventura, coautor del libro desempeñó un papel activo en el área educativa del gobierno militar a partir del ejercicio del cargo de Subsecretario de Educación. 
cional del terror, la dádiva y la mentira. Aquella etapa se habría caracterizado además por la ola de "inmoralidad y decadencia" que signó a los estrados oficiales. La perspectiva fuertemente crítica del gobierno de Perón se extiende también a su actuación en la época de su exilio y, en particular, durante su tercer gobierno. Perón pasa a convertirse durante estos años, según estos autores, en un auténtico promotor del terrorismo.

Una parte especial de esta obra está dedicada a analizar los efectos de la política ejercida por el tercer gobierno peronista sobre el sistema educativo y, en particular, sobre la Universidad. Un pasaje especial se dedica aquí al "desquiciamiento" de la educación argentina que tuvo lugar bajo el ministerio de Jorge Taiana entre mayo de 1973 y agosto de 1974. En la obra se denuncia la "acción deleterea" ejercida por el "marxismo" en los claustros de "nuestra patria". En aquellos meses "el caos", se enseñoreó en las universidades prosiguiendo las "bandas marxistas su labor de destrucción". De este modo, las Universidades habrían sufrido, durante esta etapa, la influencia nefasta del "marxismo comunista"7.

\section{El orden en el país y la Universidad: la impronta occidental y católica}

Como se acaba de señalar, la filiación y los estrechos lazos con la comunidad católica en sus vertientes más conservadoras constituyeron un rasgo común a varios de los Rectores de las Universidades públicas durante el período estudiado $^{8}$. Por otra parte, como ha señalado Laura Rodríguez, la pertenencia al mundo católico constituyó un rasgo común no sólo a los Rectores sino también a los funcionarios y ministros de la Dictadura en líneas generales 9 . Lennon y Rodríguez Varela no constituyen una excepción al respecto. Esto puede advertirse no sólo por su participación en círculos vinculados a instituciones educativas confesionales como las ya mencionadas universidades católicas o en el caso de Lennon por su declarada pertenencia a la corporación de abogados católicos "San Alfonso María de Ligorio", sino también por la concepción del orden político y social que revelan sus discursos y que

${ }^{7}$ Romero Carranza, Rodríguez Varela y Ventura, Historia Política, 411. Como señalamos pueden verse también las ediciones anteriores que contienen escasos cambios en relación con esta versión.

${ }^{8}$ Al respecto veáse Laura Rodríguez, "La Universidad Argentina durante la última dictadura: actitudes y trayectorias de los rectores civiles (1976-1983)", Revista Binacional ArgentinaBrasil, v.3, (2014): 135-160.

${ }^{9}$ Rodríguez, Católicos y nacionalistas, 117. 
tenía una influencia decisiva en su visión del sistema educativo. En primer término debemos señalar que los pronunciamientos públicos en defensa del orden impuesto por la Dictadura, como es previsible, eran habituales en los dos, pero se encuentran con mayor frecuencia en Lennon probablemente debido a que desarrolló su gestión en la etapa de consolidación del régimen.

Las referencias a la impronta católica que debía conservar el orden social y político eran, por otro lado, habituales en los dos casos. Cuando Lennon asumió como Decano de la Facultad de Derecho y Ciencias Sociales aseguró que la etapa abierta con el "Proceso" se había caracterizado por el saneamiento moral de la república dando fin a un período de "anarquía, indisciplina y demagogia" ${ }^{10}$. En sus discursos y pronunciamientos públicos eran frecuentes las alusiones y la fundamentación de sus perspectivas con las palabras y expresiones de los Papas, en particular de Pablo VI. En diciembre de 1978 afirmó su deseo de que la UBA constituyese un "templo" que "cobijase a maestros y discípulos" enfatizando en el ejercicio de las virtudes que constituían el néctar de nuestra "civilización cristiana"11. En el acto de asunción del nuevo Decano de la Facultad de Odontología, en abril de 1979, subrayó que no había espacio para los que "venían a sembrar ideologías repugnantes a los principios consustanciales con la Argentina cristiana y democrática"12. Por otra parte, un homenaje al Prebístero Antonio Saénz, Primer Rector de la UBA, le permitía también asociar los orígenes de la Universidad con sus raíces católicas ${ }^{13}$. Puede subrayarse entonces aquí el papel central del catolicismo como articulador ideológico de las relaciones entre las jerarquías universitaria y militar. En este sentido, cabe destacar que diversos autores han subrayado el peso ideológico del nacionalcatolicismo entre las principales figuras vinculadas con la Dictadura. Como ha señalado Loris Zanatta, las autoridades militares procuraban afirmar un sistema educativo consistente con los valores de la "civilización occidental y cristiana" asumiendo así el vínculo indisoluble entre catolicismo e identidad nacional siendo lo nacional

10 "Discurso del Dr. Lucas Lennon al asumir el cargo de Decano de la Facultad de Derecho y Ciencias Sociales", 28 de marzo de 1977, en Rectores, Lucas J. Lennon, Documento N 2.

11 "Palabras de Lucas J. Lennon con motivo de la entrega de medallas recordativas al personal, 15 de diciembre de 1978", en Rectores, Lucas J. Lennon, Documento N 10.

12 "Asumió su cargo el Decano de la Facultad de Odontología", 20 de abril de 1979 en Rectores, Lucas J. Lennon, Documento N 14

13 "Homenaje al Prebístero Saénz. Discurso del Rector Doctor Lucas J. Lennon, 1. de septiembre de 1980". En Rectores, Lucas J. Lennon, Documento 41. Debe recordarse aquí que la UBA fue fundada en 1821 y, aunque su organización se efectivizó en un contexto de conflicto con las autoridades religiosas, sus rectores, hasta 1852 fueron todos sacerdotes. 
definido a partir de la primacía de la religión católica ${ }^{14}$. Otros autores, como Martín Obregón y Emilio Mignone, han llamado la atención sobre la participación decisiva de sectores relacionados con círculos católicos conservadores tanto en las áreas del gobierno vinculadas con la educación en todos sus niveles como en la justicia. En los procesos de formación del gobierno y selección de sus autoridades los militares, como ha destacado Zanatta, consultaban periódicamente con miembros del Episcopado, la Universidad Católica y el Consejo Superior de Educación Católica, responsable este último de la administración de gran parte de las escuelas confesionales. La mayoría de los Ministros de Educación, por otra parte, eran reconocidos públicamente como católicos practicantes ${ }^{15}$.

Debe señalarse también que las reflexiones de Rodríguez Varela y Lennon sobre la Universidad incluían referencias permanentes a la necesidad de poner en marcha una formación que equilibrase aspectos científicos y humanistas. Para los dos, la casa de altos estudios era el lugar por excelencia de formación de las élites dirigentes. Lennon, en una reunión con alumnos ingresantes, subrayó que la función de la Universidad consistía en instruir individuos cabales y amantes de la verdad y la justicia y que la institución debía priorizar la formación de hombres cultos, sabios, virtuosos, que además debían rendir un fervoroso culto a la patria ${ }^{16}$. Para Rodríguez Varela la formación universitaria debía estar signada por el humanismo de la cultura occidental. En febrero de 1982 propuso a los estudiantes de derecho formarse en base a una visión humanista del orden jurídico fundado en las más nobles vertientes del pensamiento occidental. Este humanismo debía estar, además, teñido por la tradición cristiana. Otro aspecto al que ambos otorgaban un papel relevante en el espacio de la formación universitaria era el del deporte. El estímulo a la actividad deportiva se entendía en el contexto de una política tendiente a lograr el desarrollo armonioso y pleno de la personalidad. En Noviembre de 1981, Lennon señaló que su gestión había presenciado los más

${ }^{14}$ Fenómenos de similares características tuvieron lugar en esta misma época en la Universidad Nacional de Córdoba, acentuados en este caso por el origen jesuítico de la Universidad, al respecto puede verse Marta Philp, "La Universidad Nacional de Córdoba y la formación de las almas”, Universidad Nacional de Córdoba, Cuatrocientos años de Historia, coord. Daniel Saur y Alicia Servetto (Córdoba, Universidad Nacional de Córdoba, 2013): 275-296.

${ }^{15}$ Loris Zanatta, La larga agonía de la Nación Católica (Buenos Aires: Sudamericana, 2015). Véase también sobre las relaciones entre la Iglesia Católica y la Dictadura, Emilio Mignone Iglesia y Dictadura (Bernal: Universidad Nacional de Quilmes, 1999) y Martín Obregón, Entre la cruz y la espada (Bernal: Universidad Nacional de Quilmes, 2005).

16 "Alumnos ingresantes se reunieron con el Rector de la Universidad de Buenos Aires, 21 de mayo de 1980". En Rectores, Lucas J. Lennon, Documento N 37. 
altos índices de participación estudiantil en actividades deportivas y subrayó como, ante las convocatorias del Rectorado, los estudiantes habían dado una respuesta numerosa y disciplinada ${ }^{17}$.

Otro aspecto común a ambos era la defensa de la idea de la Universidad como una comunidad jerárquicamente organizada. En este sentido rechazaban la posibilidad de que la Universidad argentina fuese cogobernada por profesores, egresados y alumnos como lo había estado durante gran parte de su historia a partir de las acciones del movimiento reformista de 1918. El rechazo explícito a la tradición reformista constituyó un aspecto común a la mayoría de los funcionarios de la Dictadura con responsabilidades en el campo de la educación superior. El Subsecretario de Educación, Eduardo Ventura, de quien dependieron además ambos Rectores, señaló en julio de 1979 en referencia a esta tradición: "Queremos una Universidad y no un soviet tumultuoso de profesores, alumnos y egresados"18. Estos criterios y orientaciones dominaban, como es obvio, también en el ordenamiento legal que el régimen sancionó en 1980 para la Universidad. La ley 22202 establecía la designación de los Rectores por el Poder Ejecutivo y de los Decanos por el Ministerio de Cultura y Educación a propuesta de estos. La ley aseguraba, de todos modos, el nombramiento de los profesores por concurso y prohibía a los miembros de los cuerpos directivos de las casas de estudios ejercer cargos en partidos políticos o en gremios o formular declaraciones públicas en esos ámbitos ${ }^{19}$.

\section{La UBA en el contexto de las políticas universitarias nacionales}

La política universitaria de la Dictadura tuvo dos ejes fundamentales: el primero de ellos fue el férreo control ideológico y político dentro de los claustros. La Dictadura llevó a cabo cesantías masivas de docentes y estudiantes, cerró de manera provisoria y, en algunos casos, definitiva, carreras enteras sobre todo en las áreas artísticas y de humanidades e impuso mecanismos, procedimientos y prácticas represivas dentro de las universidades. El segundo eje estuvo centrado en la limitación del crecimiento de la matrícula que había aumentado en forma acelerada desde los años cincuenta ${ }^{20}$. Como ha

17 "Lucas Lennon. Palabras al inaugurar la pileta olímpica en el campo de deportes de Ciudad Universitaria. 18 de noviembre de 1981" en Rectores, Lucas J. Lennon, Documento N 60.

18 "La pelea por la nueva Universidad", en Confirmado, 6 (Julio de 1979), 14-18.

${ }^{19}$ Sobre esta ley Soprano y Rodríguez, "La política Universitaria", en Nuevo Mundo, Mundos Nuevos (2009).

${ }^{20}$ En 1947 había poco más de 50 mil estudiantes universitarios, en 1957 ascendían ya a 
señalado Augusto Pérez Lindo se trataba de conformar un sistema universitario más pequeño, "eficaz" y controlable ${ }^{21}$. Debemos recordar además que el régimen militar procuró avanzar en el proceso de institucionalización del sistema universitario. Las dos figuras estudiadas aquí manifestaron explícitamente su apoyo a la ya mencionada ley sancionada en abril de 1980 e insistieron, sobre todo, en la necesidad de normalizar el claustro de profesores invocando entonces su preocupación por avanzar en los concursos docentes.

De todas formas, las intervenciones públicas de Rodríguez Varela muestran, a diferencia de las de Lennon, una clara preocupación por recuperar dimensiones específicamente académicas y científicas de la vida universitaria más allá de la impronta fuertemente católica que impregna su discurso. Esta preocupación puede advertirse en diferentes planos, pero se hizo evidente sobre todo a la hora de presentar a los nuevos Decanos que se hicieron cargo de las diferentes Facultades al comenzar su período como Rector. El perfil académico de los nuevos funcionarios aparece así más claramente delineado que en el caso de los de la época de Lennon donde lo fundamental estaba limitado al aporte a la construcción de un nuevo orden político en la casa de estudios o al hecho de haber renunciado o haber sido desplazado de la institución a partir de mayo de 1973 en los primeros tramos del tercer gobierno peronista.

Por otra parte, las discusiones relativas a la matrícula estudiantil, la forma de selección de los estudiantes o los problemas relacionados con las condiciones de estudio en la UBA aparecen con frecuencia a lo largo de la gestión de Lennon. El tema se hizo presente, de manera habitual, en las reuniones que celebraba periódicamente como Rector con núcleos reducidos de estudiantes de distintas facultades seleccionados, por lo general, sobre la base de sus destacadas calificaciones. Si bien como es previsible en los documentos oficiales no se muestran disidencias de fondo entre el Rector y los estudiantes pueden advertirse, en repetidas oportunidades, reclamos relacionados con las condiciones de estudio. En septiembre de 1979, en una reunión con Lennon alumnos de la Facultad de Derecho sugirieron la necesi-

145 mil, en 1965 a 222 mil y en 1975 gracias a la política de apertura del ingreso llevada cabo por el tercer gobierno peronista eran ya 507 mil. En 1983 como resultado de la política de restricciones al ingreso disminuyeron hasta 416 mil. Debe tenerse en cuenta también que en 1975 la participación en la matrícula del sector privado llegaba a un 10\% del total mientras era del 19\% en 1983. Los datos en Daniel Cano, La Educación Superior en la Argentina (Buenos Aires: FLACSO, 1985), 123-124.

${ }^{21}$ Augusto Pérez Lindo, Universidad, Política y Sociedad (Buenos Aires: Eudeba, 1985), 175 y siguientes. 
dad de abrir un mayor número de cursos con sistema de promoción directa. La respuesta del Rector insistió en la necesidad de que la Universidad asegurase solamente las condiciones que permitiesen garantizar la calidad tanto de los estudios como de los graduados ${ }^{22}$.

Debe recordarse, en este contexto, que el tema del ingreso constituyó uno de los motivos que desencadenaba la presencia permanente de la cuestión universitaria en la prensa. En 1979 las autoridades de la UBA confirmaron que había 46312 inscriptos para 11050 vacantes. La cuestión del acceso era particularmente aguda en las grandes universidades, pero en ninguna de ellas la falta de vacantes era tan pronunciada como en la UBA. En la Universidad Nacional del Sur, por ejemplo, durante el mismo año se registraba la existencia de 1305 vacantes para 1438 postulantes. Incluso en Córdoba la situación no era tan grave ya que se registraban 9530 aspirantes para 4665 vacantes. En La Plata, mientras tanto, se habían inscrito 7876 estudiantes para 4600 vacantes $^{23}$.

La cuestión del ingreso constituyó uno de los ejes de controversia en la prensa sobre la política universitaria y era uno de los pocos temas sobre los que se advierten críticas abiertas al gobierno. Incluso suscitó intervenciones públicas de figuras y organizaciones de declarada filiación política, fenómeno poco usual en aquellos tiempos. Desde los principales diarios se insistió en subrayar el número elevado de jóvenes que no podrían acceder a los estudios universitarios. En La Opinión se denunciaba, en febrero de 1979, la ineficacia del sistema de ingreso: los horarios de los cursos, se aclaraba, eran "incompatibles con las actividades laborales" y se le proponía a los aspirantes “... una maratón en donde tres cuartas partes de quienes compiten en ella no lograrán ver el final”24. Ese año también Clarín registró como tres agrupaciones universitarias vinculadas con partidos políticos (Frente de Izquierda Popular, la Agrupación Universitaria Desarrollista y los Estudiantes Universitarios Justicialistas) solicitaban el ingreso abierto en la Universidad ${ }^{25}$.

22 "Alumnos distinguidos de la Facultad de Derecho y Ciencias Sociales se reunieron con el Rector de la Universidad de Buenos Aires", 5 de septiembre de 1979, en Rectores, Lucas J. Lennon, Documento N 25.

${ }^{23}$ Según Pérez Lindo en 1974 el número de estudiantes ingresantes a las universidades nacionales sumó 127606, en 1976 fueron 89952 y en 1977, 43924. En la Universidad de Buenos Aires, mientras tanto, en 1974 ingresaron 40825 y en 1977 sólo 13312. Véase Pérez Lindo, Universidad, Política y Sociedad, 181.

24 "Detalles de la Metodología que se aplica en cursos de ingreso", en La Opinión, 11 de febrero de 1979.

25 "Piden ingreso abierto en 1979", en Clarín, 8 de febrero de 1979. Véase también, “Universitarios radicales contra el examen de ingreso", en La Nación, 23 de febrero de 1979. 
Desde el Rectorado se respondió de manera ambigua y hasta cierto punto oblicua a los reclamos. Como era previsible, un régimen de estas características no encontraba necesario establecer un debate o polémica sobre estas cuestiones ni sus funcionarios se sentían forzados a abrir la discusión. De todas formas, el tema no estuvo ausente en las intervenciones de Lennon. En agosto de 1980, en una reunión en el Rotary Club, subrayó la necesidad de planificar el crecimiento destacando que la Universidad debía diferenciarse del resto del sistema de educación superior en base a la búsqueda del saber puro y desinteresado y que era necesario que los alumnos tuviesen "aptitud universitaria" y "madurez espiritual" y que estas no se deducían automáticamente de la condición de egresado de la enseñanza media. En contrapartida, en este mismo contexto insistió en más de una oportunidad en subrayar la transparencia del sistema de evaluación ${ }^{26}$.

En mayo de 1981, ante una consulta de una estudiante de la carrera de psicología, Lennon afirmó que los motivos que imponían los cupos eran fundamentalmente académicos y remitían sobre todo a limitaciones edilicias y administrativas ${ }^{27}$. La respuesta era, en algún modo significativa ya que negaba la idea de que la política limitacionista obedeciese a una determinación previa del número de profesionales que se intentaba formar motivada a su vez en un estudio de las necesidades del país. Es llamativa, por otra parte, la escasa atención que tanto Lennon como Rodríguez Varela prestaron a otra dimensión de la política universitaria clave de aquellos años como fue la imposición de los aranceles a los estudios de grado efectuada desde 1981 aspecto que, prácticamente, no aparece en sus discursos ${ }^{28}$.

Otra dimensión de la política oficial que tanto Lennon como Rodríguez Varela atendieron fue el de los esfuerzos por institucionalizar el régimen universitario que procuró llevar a cabo el gobierno dictatorial. Lennon subrayó en varias oportunidades la necesidad de avanzar en ese sentido. En febrero de 1979, al poner en funciones al Decano de la Facultad de Ciencias Económicas, Cayetano Licciardo, destacó que en un futuro próximo habría

${ }^{26}$ Lucas Lennon, "La importancia de la Universidad en el futuro de la República”, agosto de 1980, en Rectores, Lucas J. Lennon, Documento 39.

27 "Recibió el rector a alumnos ingresantes altamente calificados", 6 de mayo de 1981, en Rectores, Lucas J. Lennon, Documento 49.

${ }^{28}$ Debe notarse aquí que las universidades fueron aranceladas hasta principios de la década de 1950. El primer peronismo estableció la gratuidad de la enseñanza universitaria, aunque en algunos casos y por períodos breves las universidades volvieron a ser aranceladas. La imposición de los aranceles en 1981 constituyó uno de los ejes de resistencia del movimiento estudiantil en la última fase de la Dictadura. Véase Pablo Buchbinder, Historia de las Universidades Argentinas (Buenos Aires: Sudamericana, 2010), 33 y siguientes. 
que normalizar los claustros de acuerdo con la nueva ley universitaria y volvió sobre la cuestión en forma insistente en varias oportunidades ${ }^{29}$. Pero, como es previsible, el tema de la institucionalización y normalización está mucho más presente en los pronunciamientos públicos de Rodríguez Varela que ejerció su cargo, en gran parte, durante los últimos meses de la Dictadura y ya cuando estaba en marcha el proceso de transición a la democracia.

En base a la ley mencionada, la UBA sancionó en el año 1981 nuevos estatutos. En diciembre de ese año, el Rector insistió en señalar que la autonomía académica, la autarquía administrativa y la libertad de cátedra sólo tenían sentido en función de los altos fines de la vida universitaria. En ese contexto destacó también la necesidad de avanzar en los concursos de profesores en el marco del mismo proceso de institucionalización de la casa de altos estudios. Rodríguez Varela aspiraba a que la Universidad de Buenos Aires fuese el primer ejemplo de un proceso exitoso de normalización que sentaría las bases de una nueva Universidad con continuidad en la etapa democrática. Con ese objetivo, además de sancionar un nuevo estatuto, impulsó un proceso masivo de concursos -a partir de un reglamento que favorecía a los profesores interinos- que generó una intensa oposición configurando uno de los aspectos más debatidos de la situación universitaria en la prensa. A partir de abril de 1982, Rodríguez Varela procuró articular su política de normalización universitaria con el proceso de apertura institucional promovido desde el gobierno.

De este modo, el ex Ministro de Justicia defendió, con diversos argumentos, los concursos -que fueron fuertemente cuestionados- destacando, sobre todo, la solvencia intelectual y científica de los jurados. Negó en este contexto que la normalización se llevase a cabo bajo una definida tonalidad política señalando que no existían casos de discriminación política e ideológica. También argumentó que los concursos de esta etapa eran más abiertos y transparentes que los efectivizados entre 1956-1958 considerada aún entonces el inicio de una verdadera "edad de oro" de la Universidad Argentina ${ }^{30}$. La oposición a los concursos fue compartida por diversos actores tanto políticos como universitarios aglutinando entonces desde agrupaciones gremiales estudiantiles hasta colegios profesionales. Un reconocido y prestigioso especialista en educación, Héctor Félix Bravo, objetó, en particular, que el Reglamento de Concursos considerase como "antecedente relevante el desempeño del aspirante en el cargo al tiempo de llamarse a concurso" y

29 "Palabras de L.J. Lennon en la Facultad de Ciencias Económicas. 7 de febrero de 1979", en Rectores, Lucas J. Lennon, Documento N 13.

30 "La inobjetabilidad de los concursos", en La Voz, 1 de noviembre de 1982. 
también la facultad del Jurado para eximir de la prueba de oposición a aspirantes que ya se desempeñasen como profesores interinos ${ }^{31}$.

Pero debe señalarse que la política de concursos fue objeto de debate y que la postura de Rodríguez Varela así como fue fuertemente cuestionada, entre otras figuras por el ya mencionado ex Rector y Presidente del Centro de Ingenieros, Alberto Constantini o por Antonio Troccoli, uno de los principales dirigentes de la UCR también fue respaldada por instituciones como el Colegio de Abogados de Buenos Aires, la Corporación de Abogados Católicos o por figuras reconocidas del mundo académico y científico como Luis F. Leloir u Horacio Sanguinetti. El primero de ellos, un prestigioso científico galardonado con el Premio Nobel de Química en 1970, los defendió basándose en su propia participación como jurado y el segundo, un reconocido abogado identificado con la tradición reformista, señaló que no era posible manipularlos y que su desconocimiento generaría serías demoras en los futuros procesos de normalización ${ }^{32}$.

\section{La Universidad en sus vínculos con el mundo científico, político y cultural}

Tanto Lennon como Rodríguez Varela fueron figuras que incursionaron, de manera frecuente, en el ámbito público. Dictaban conferencias sobre problemas universitarios, participaban en actos oficiales e intervenían expresando su concepción sobre el sistema de educación superior en distintos espacios institucionales. En este sentido, procuraron siempre ocupar un espacio destacado dentro de la vida pública y sus expresiones figuraron, a menudo, en un lugar relevante en la prensa periódica. A través de estos pronunciamientos fijaban posición en torno a cuál debía ser la orientación general de la actividad universitaria y además establecían instancias de vinculación y formas de aproximación a figuras e instituciones prestigiosas del estado y la sociedad civil.

Obviamente, en ambos casos, se puede advertir el intento de construir una relación privilegiada con las Fuerzas Armadas ${ }^{33}$. Lennon inauguró, en

${ }^{31}$ La opinión de Bravo en "Concursos a medida”, en Redacción, N 117, noviembre de 1982, pp 19.

${ }^{32}$ Veanse las críticas de Constantini en "Universidad a oscuras", La Nación, 8 de agosto de 1982, las opiniones de Leloir, en "Leloir defendió los concursos universitarios", en El Litoral, 30 de octubre de 1982 y las de Sanguinetti en "Sanguinetti: los concursos universitarios no se pueden manipular", en Convicción, 26 de agosto de 1982.

${ }^{33}$ Los vínculos de las dos figuras estudiadas aquí con altos jefes del ejército y funcionarios de la dictadura continuaron ya en tiempos de la democracia. Lennon fue abogado de Reynal- 
dos oportunidades, ciclos de cursos de Defensa Nacional en el ámbito de la Universidad. En este marco destacó la necesidad de que las dos instituciones construyeran vínculos estrechos y que los integrantes de ambas confraternizasen y compartiesen espacios de formación. En el caso de Rodríguez Varela, el período de la Guerra de Malvinas ofreció una oportunidad para avanzar en los vínculos entre ambas. Rodríguez Varela, asiduamente, a través de la prensa colaboró, sobre todo, a la hora de justificar la acción y conducta del gobierno militar en relación con la intervención que comenzó el 2 de abril. Pero también participó en la inauguración de un programa en la Facultad de Medicina denominado "Organización Sanitaria Militar en el Teatro de Operaciones" y que tenía como propósito instruir a los inscriptos en los modos de conformar el registro de voluntarios de enfermería, de médicos residentes, de estudiantes de medicina y de personas provenientes de instituciones sanitarias en general. Se trataba entonces de la conformación, no de un curso de primeros auxilios, sino, fundamentalmente, de uno de organización administrativa y de derivación de pacientes destinado a brindar auxilio y apoyo en el marco del conflicto ${ }^{34}$. En este contexto se esperaba que la Facultad de Medicina cumpliese un papel esencial.

En el caso de Lennon uno de los modos particulares de vincular a la Universidad con figuras e instituciones reconocidas del mundo intelectual y cultural fue la concesión de los Doctorados Honoris Causa. En este sentido, debe señalarse que se dieron 17 Doctorados Honoris Causa en la UBA durante todo el período de la Dictadura y que 12 de ellos se otorgaron bajo su gestión $^{35}$. Es particularmente interesante observar quienes fueron los que recibieron dicha distinción. Además, también pueden advertirse algunos rasgos significativos de su pensamiento a partir de sus discursos en los actos de otorgamiento de los doctorados.

Estos actos de entrega de distinciones servían también para reafirmar líneas de acción y establecer posicionamiento frente a controversias científicas y políticas. Durante su Rectorado se otorgaron títulos de Doctor Honoris Causa, entre otros, al Rey Juan Carlos de España, al arquitecto japonés Kenzo Tange, al Ministro de Relaciones Exteriores del Japón, a los médicos franceses René Truhaut y Jerome Lejeune, al médico italiano Luigi Gedda, a

\footnotetext{
do Bignone y Rodríguez Varela de Jorge Rafael Videla, ambos presidentes durante el período dictatorial. Defendieron a ambos en las causas por violación de derechos humanos.

${ }^{34}$ En "Organización sanitaria militar en el Teatro de Operaciones. Se inauguró hoy un curso especial en la Facultad de Medicina", 19 de mayo de 1982, en Rectores, Alberto Rodríguez Varela, Documento N 17.

${ }^{35}$ Véase La causa de los Doctores, (Buenos Aires: Eudeba, 2001): 77-81.
} 
los intelectuales españoles Julián Marías y Demetrio Ramos Pérez y al Ministro de Justicia del Uruguay Fernando Bayardo Bengoa. También ofreció una entusiasta recepción al Director General de la Unesco, al escritor Germán Arciniegas y realizó un sentido homenaje al jurista Roberto Repetto ${ }^{36}$.

De las palabras pronunciadas por Lennon en estos actos es posible advertir la voluntad de afianzar vínculos con figuras e instituciones con evidente afinidad con sus concepciones políticas e ideológicas. Obviamente también aquí son claros los lazos con personajes vinculados estrechamente con el mundo educativo y científico católico. En este sentido, la concesión del Doctorado Honoris Causa al Dr. Luigi Gedda conserva un significado especial. Gedda era especialista en Genética, pero Lennon destacó en el discurso de otorgamiento de la distinción sobre todo su protagonismo político y cultural en la Italia de posguerra. Subrayó como Pío XI le había encargado la organización y Dirección de la Juventud Italiana de Acción Católica, en primer término, e insistió en su papel como organizador de las fuerzas católicas para detener al "comunismo" y su rol, luego, como Presidente de la Acción Católica Italiana. El estrecho vínculo con el mundo católico también puede percibirse en el caso de Jerome Lejeune, un prestigioso genetista que era, desde 1974, miembro de la Academia Pontificia de las Ciencias y que fue incluso designado, posteriormente, Presidente Vitalicio de la Academia Pontificia para la Vida.

Así, puede advertirse el interés de Lennon por distinguir a figuras del ámbito científico y médico especialistas en temas de Genética y caracterizados por su vínculo con el mundo católico. Pero también pueden destacarse otros aspectos. En principio, su voluntad de relacionarse con la tradición hispánica, también en su vertiente católica y franquista. Las palabras pronunciadas en los actos de distinción del Rey Juan Carlos, de Julián Marías y del historiador Demetrio Ramos Pérez, -un catedrático de la Universidad de Valladolid conocido por su credo franquista y falangista- revela claramente esa voluntad. Por otra parte, el homenaje al jurista Roberto Repetto, miembro de la Corte Suprema en tiempos del golpe del 6 de septiembre de 1930 le servía para subrayar los fundamentos jurídicos que podían sostener las intervenciones militares e interrupciones del orden constitucional ${ }^{37}$. La distinción al

${ }^{36}$ Véase especialmente, "El Rey de España, don Juan Carlos I, recibe el título de Doctor "Honoris Causa" de la Universidad de Buenos Aires", 29 de noviembre de 1978, y "Entrega del título de Doctor "Honoris Causa" al profesor Ramos Pérez", 29 de mayo de 1980. En Rectores, Lucas J. Lennon, Documentos N 6 y 38.

${ }^{37}$ La Corte Suprema que integraba Repetto dio aval jurídico al golpe de estado milita del 6 de septiembre de 1930. 
Ministro de Justicia del Uruguay, también entonces bajo un gobierno militar, Fernando Bayardo Bengoa, era útil para poder reafirmar lazos con un régimen afín al argentino ${ }^{38}$.

\section{Reflexiones finales}

El análisis de las trayectorias, las intervenciones públicas y discursos de Lucas Lennon y Alberto Rodríguez Varela en el período de su Rectorado al frente de la UBA posibilita obtener una primera aproximación a la situación de la casa de estudios en tiempos de la Dictadura. Este estudio permite advertir, como era previsible, la identificación lisa y llana de ambas figuras con los lineamientos generales de política impuestos por el régimen militar y en particular de su política educativa y universitaria, luego de una primera etapa signada por disidencias menores entre las autoridades del Ministerio y el gobierno de la UBA.

Los discursos posibilitan advertir la profunda impronta católica que impregnaba la concepción del orden político social y educativa de ambas figuras y nos permiten ver como el ámbito universitario constituyó un campo privilegiado en el que se articularon los vínculos entre las autoridades militares y sectores integristas del catolicismo. La Universidad debía sostenerse en un orden jerárquico revelando así, ambos personajes, su profunda hostilidad a las tradiciones universitarias reformistas provenientes de 1918. Esta perspectiva jerárquica constituyó uno de los ejes centrales con el que ambos percibieron al mundo universitario.

Los dos intentaron establecer una relación privilegiada con las fuerzas armadas. En el caso de Lennon fue fundamental la organización de cursos de defensa en el ámbito universitario al que concurrían tanto alumnos de la institución como oficiales de las Fuerzas Armadas. En el de Rodríguez Varela esta voluntad se exteriorizó particularmente en tiempos de la guerra de Malvinas, entre otros aspectos a partir de la organización de un curso particular para voluntarios en la Facultad de Medicina.

De todas formas, es posible advertir entre ambas diferencias de matiz en el discurso. Mientras Lennon enfatizó habitualmente en todos aque-

${ }^{38}$ Un análisis del uso del Doctorado Honoris Causa en la Universidad Nacional de Córdoba durante un período anterior al estudiado aquí en Marta Philp y Eduardo Escudero, "Dos Doctores Honoris Causa de la Universidad Nacional de Córdoba: una historia de los vínculos entre Universidad y Política" en Universidad Nacional de Córdoba, 400 años de Historia, coord. Daniel Saur y Alicia Servetto (Córdoba: Universidad Nacional de Córdoba, 2013), 211-229. 
llos aspectos relacionados con la construcción de un orden en la Universidad consistente con los objetivos generales de la Dictadura y signado por el orden y la jerarquía, la gestión de Rodríguez Varela se caracterizó por una mayor preocupación por los aspectos académicos y científicos y por la institucionalización y normalización de la casa de estudios. Estas diferencias se explican, en principio, aunque no únicamente, por los distintos momentos históricos en los que cada uno debió desarrollar su gestión.

Puede señalarse también, a modo de conjetura, que, mas allá de su voluntad de pensar el orden social y político y a la Universidad en base a parámetros relacionados con el orden católico no se advierte en ninguno de los dos casos la intención de avanzar en un proceso de politización o de adoctrinamiento intenso en el orden universitario en su conjunto. Sus discursos no parecen haber cristalizado en acciones de formación masiva a través de cursos o asignaturas específicas. Más bien lo que puede advertirse es cierta voluntad de asegurar la "despolitización" como estrategia hacia el interior de la Universidad. Esta línea de acción es, en alguna medida, consistente con las políticas de la dictadura tendientes a asegurar el orden social a partir de la desmovilización, represión y control de las fuerzas políticas y sociales. Como ha señalado Augusto Pérez Lindo se trataba de un gobierno que no procuraba despertar adhesiones sino garantizar la obediencia a sus imposiciones generales. En este marco, creemos, deben leerse las estrategias discursivas de las figuras aquí analizadas ${ }^{39}$.

\section{Bibliografia}

Aguila, Gabriela. "La Universidad Nacional de Rosario en Dictadura", en Polhis, N 17 (2015): 146-178.

Buchbinder, Pablo. Historia de las Universidades Argentinas, Buenos Aires: Sudamericana, 2005.

Canelo, Paula. El proceso en su laberinto, Buenos Aires: Prometeo, 2008.

CANo, DANIEl. La educación superior en la Argentina, Buenos Aires: Flacso, 1985.

Jordan, AlBerto. El Proceso, Buenos Aires: Emecé Editores, 1983.

Kaufmann, Carolina. Dictadura y Educación, Buenos Aires: Miño y Dávila, 2011.

LANZilotta, Sofía L. y CASTRo Feijóo. Lucía, Justicia y Dictadura, Buenos Aires: Ediciones del CCC, 2014.

\footnotetext{
${ }^{39}$ Augusto Pérez Lindo, Universidad, Política y Sociedad, 181.
} 
Mignone, EmiLio. Iglesia y Dictadura, Bernal: Universidad Nacional de Quilmes, 1999.

Novaro, Marcos y Palermo, Vicente. La Dictadura Militar 1976/1983, Buenos Aires: Paidós, 2001.

Pallma, Sara. "Un año en la Universidad", en Perspectiva Universitaria, N 2, (1977), 61-85.

OBREGón, MARTín. Entre la cruz y la espada, Bernal: Universidad Nacional de Quilmes, 2005.

Perel, Pablo. Raíces, Eduardo y Perel, Martín, Universidad y Dictadura, Buenos Aires: Ediciones del CCC, 2006.

Quiroga, Hugo. El tiempo del proceso, Rosario: Homo Sapiens, 2006.

PÉREz Lindo, Augusto. Universidad, política y sociedad, Buenos Aires: Eudeba, 1985.

Philp, Marta y Escudero, EduARdo. "Dos doctores honoris causa de la Universidad Nacional de Córdoba: una lectura de los vínculos entre Universidad y Política"en Saur, Daniel y Servetto, Alicia, (coordinadores), Universidad Nacional de Córdoba, 400 años de Historia, Córdoba: Universidad Nacional de Córdoba, 2013, 211-229.

PhilP, MARTA. "La Universidad Nacional de Córdoba y la formación de las almas", en Saur, Daniel y Servetto, Alicia, (coordinadores), Universidad Nacional de Córdoba, 400 años de Historia, Córdoba: Universidad Nacional de Córdoba, 2013, 275-296.

PineAU, PABlo. "Reprimir y discriminar. La educación en la última dictadura cívico-militar (1976-1983), en Educar em Revista, 51, (2014) 103-122.

RodríGUEZ, LAURA. "La Universidad Argentina durante la última dictadura: actitudes y trayectorias de los rectores civiles (1976-1983)", Revista Binacional Argentina-Brasil, v.3, (2014), 135-160.

RODRÍGUEZ, LAURA. Católicos, nacionalistas y políticas educativas en la última Dictadura, Rosario: Prohistoria, 2011.

Rodríguez, LAURA y Soprano, GERMÁn. "La política universitaria de la Dictadura Militar en la Argentina: proyectos de reestructuración del sistema de educación superior", en Nuevo Mundo-Mundos Nuevos, 2009, consultable en nuevomundo.revues.org/56023.

Romero Carranza, Ambrosio, Rodríguez Varela, Alberto y Ventura, EduARdo. Historia Política y Constitucional Argentina, Buenos Aires: AZ editores, 1977.

Romero Carranza, Ambrosio, Rodríguez Varela, Alberto y Ventura, EduARDo. Historia Política y Constitucional Argentina, Buenos Aires: Az editores, 1983. 
Romero Carranza, Ambrosio, Rodríguez Varela, Alberto y Ventura, EduARdo. Historia Política y Constitucional Argentina, Buenos Aires: AZ editores, 1991.

Romero Carranza, Ambrosio, Rodríguez Varela, Alberto y Ventura, Eduardo. Historia Política y Constitucional Argentina, Buenos Aires, Círculo Militar, 1992.

Suriano, JuAn (Director). Dictadura y Democracia. Buenos Aires: Sudamericana, 2005.

Tedesco, Juan Carlos, Braslavsky, Cecilia y Carciofi, Ricardo. El proyecto educativo autoritario 1976-1983, Buenos Aires: FLACSO, 1985.

ZANATTA, Loris. La larga agonía de la Nación Católica, Buenos Aires: Sudamericana, 2015. 